\title{
Use of fat-suppressed T2-weighted sagittal images after infusion of excess saline into the subarachnoid space as a new diagnostic modality for cerebrospinal fluid hypovolemia: technical note
}

\author{
Eiichi Nakai, MD, Mitsuhiro Takemura, MD, Motonobu Nonaka, MD, Yu Kawanishi, MD, \\ Noritaka Masahira, MD, and Tetsuya Ueba, MD \\ Department of Neurosurgery, Kochi Medical School, Kochi University, Kochi, Japan
}

The diagnosis of CSF hypovolemia remains controversial. The primary diagnostic factor relies on confirmation of leakage of the CSF based on reduced spinal fluid pressure. Determining the specific leakage site is the most important issue for effective treatment but remains a difficult task. Although CT myelography, radioisotope cisternography, and MRI are commonly performed in the diagnosis of CSF hypovolemia, these techniques can rarely identify the precise leakage site. Therefore, an epidural blood patch is performed in the lumbar spine in many cases.

This study reports a new diagnostic modality that can help to confirm the leakage site. Fat-suppressed T2-weighted sagittal images were compared before and after the infusion of $20 \mathrm{ml}$ of saline into the subarachnoid space of the lumbar region to detect the specific leakage site with high probability. Three patients were successfully treated by the epidural blood patch based on data obtained with the new diagnostic modality. Two patients were treated in the cervical region and 1 in the lumbar region. The use of fat-suppressed T2-weighted sagittal images after saline infusion could be a relevant diagnostic modality compared with images obtained by CT myelography, radioisotope cisternography, and ordinary MRI to achieve accurate diagnosis and effective treatment of patients with CSF hypovolemia.

http://thejns.org/doi/abs/10.3171/2015.2.JNS142746

KEY WORDS cerebrospinal fluid hypovolemia; epidural blood patch; fat-suppressed T2-weighted sagittal image

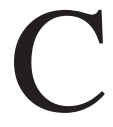
EREBROSPINAL fluid hypovolemia (CSFH) is often caused by persistent CSF leakage. CSFH usually presents with orthostatic headache, but other symptoms can be present such as nausea, vomiting, dizziness, and neck pain. The incidence of CSFH is approximately 5 per 100,000 individuals, and it occurs in women twice as often as in men. Although the precise etiology of CSFH is unknown, the most commonly proposed pathophysiological mechanism includes underlying structural weakness of the dura, leading to rupture. The most common sites for CSF leaks are the lower cervical and upper thoracic spine. ${ }^{3}$

The diagnosis of CSFH is typically established by CT myelography (CTM) or radioisotope cisternography (RIC). ${ }^{1}$ However, even with the help of various imaging modalities, it is difficult to localize the actual leakage site. The effect of the epidural blood patch (EBP) becomes unstable if the actual leakage site is unknown. Therefore, finding the actual leakage site is a critical factor for successful treatment. Here, we propose a possible new modality to resolve this issue: obtaining fat-suppressed T2weighted sagittal images (FST2WSIs) before and after a saline infusion by spinal tap.

The symptoms of 1 of the CSFH patients were temporarily ameliorated by the infusion of $10 \mathrm{ml}$ of iohexol (Omnipaque 240) in the CTM. A few hours later, the symptoms returned, but were abated again by the repeated infusion of $10 \mathrm{ml}$ of saline. Because the saline infusion by spinal tap was very effective in resolving the symptoms within

ABBREVIATIONS CSDH = chronic subdural hematoma; CSFH = CSF hypovolemia; $C$ TM = CT myelography; EBP = epidural blood patch; FST2WSI = fat-suppressed

T2-weighted sagittal image; $\mathrm{RIC}$ = radioisotope cisternography.

SUBMITTED December 3, 2014. ACCEPTED February 3, 2015.

INCLUDE WHEN CITING Published online September 18, 2015; DOI: 10.3171/2015.2.JNS142746. 


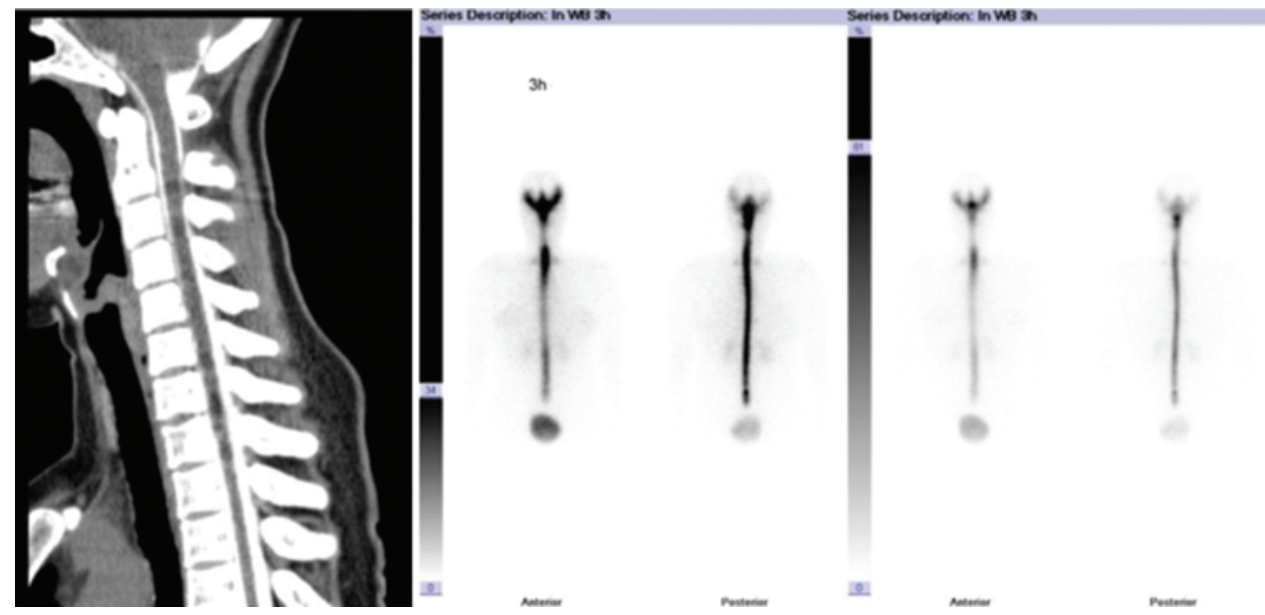

FIG. 1. Left: Leakage was not observed in the sagittal CTM. Right: Leakage was not observed in the sagittal RIC. Figure is available in color online only.

only a few hours, we determined the optimal infused saline volume to be $20 \mathrm{ml}$ while performing pressure measurements. The leakage site was successfully localized by obtaining FST2WSIs after saline infusion. This case motivated us to continue to explore this modality in CSFH treatments. Here, we describe our experience thus far.

\section{Methods}

Three patients ( 3 women; mean age 42.7 years, range 18-72 years) with CSFH, who were diagnosed based on low CSF pressure, were treated with the aid of FST2WSIs following saline infusion. The lumbar puncture was performed at L4-5, which were identified from the Jacoby's line at the lateral position. Once low CSF pressure was confirmed, infusion of saline was started. The CSF pressure was remeasured after injection of $10 \mathrm{ml}$ of the saline to confirm that there was no excessive rise in CSF pressure. After confirming that the CSF pressure was within normal limits, we infused another $10 \mathrm{ml}$ of saline. All patients reported the temporary relief from headache. FST2WSIs were obtained 3-6 hours after the saline infusion. We estimated that 3-6 hours is sufficient time for CSF infiltration before the patients complained of headache again. The optimal time after infusion should be determined to achieve a peak signal in the next series. The EBP was then performed with injection of autologous blood obtained from the patient's right brachial vein.

We treated the patients with fluoroscopically guided placement of an EBP and followed up with subsequent spinal CT scans. With the patient in a lateral position, a $22-G$ needle was inserted in the epidural space at the target level under fluoroscopic guidance, using the air lossof-resistance technique. The injection was stopped when the patient reported increased pressure sensation. Infection, iatrogenic $\mathrm{CSFH}$, and raised pressure after injection of saline are potential risk factors. In our cases, none were reported because our number of patients was small.

Fat-suppressed T2-weighted sagittal images were obtained using the following parameters: TE $100 \mathrm{msec}$, TR $3500-4000$ msec, neck $28 \mathrm{~cm}$, thorax $28 \mathrm{~cm}$, lumbar 28 $\mathrm{cm}$ field of view (GE Signa Excite HDx1.5T).

\section{Illustrative Cases Case 1}

A healthy 38-year-old woman was referred to our hospital with a 3-month history of progressively worsening headache and neck pain. Her CSF pressure was not measurable at presentation. The leakage site was not identified by CTM or RIC (Fig. 1). The leakage site was identified in C1-2 as a high-intensity area in an FST2WSI after saline infusion (Fig. 2 right). Using a sterile technique, $18 \mathrm{ml}$ of autologous blood was injected directly into the epidural space without eliciting any back pain or radicular symptoms. The patient reported complete symptomatic relief without any recurrence at follow-up of 2 months.

\section{Case 2}

An 18-year-old woman was referred to our hospital with a 1-month history of headache and neck pain after a traffic accident. Her CSF pressure was $13.5 \mathrm{~cm} \mathrm{H}_{2} \mathrm{O}$ at presentation, and had decreased to $9 \mathrm{~cm} \mathrm{H}_{2} \mathrm{O}$ upon secondary examination. The leakage site was not identified by RIC (Fig. 3 right), but was identified in the lower lumbar spine by CTM (Fig. 3 left). An FST2WSI obtained after saline infusion also identified the leakage site in the lower
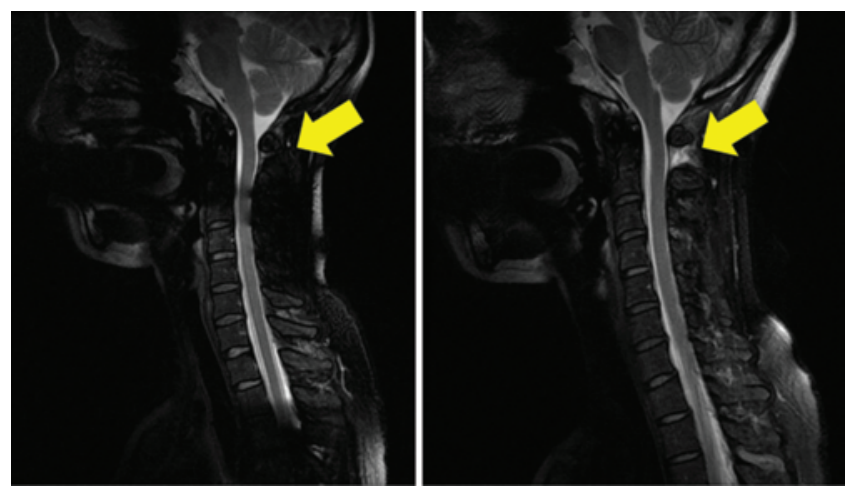

FIG. 2. Left: No finding was observed in the sagittal cervical MR image before injection (yellow arrow). Right: Leakage was observed between $\mathrm{C}-1$ and $\mathrm{C}-2$ in the sagittal cervical MR image after injection (yellow arrow). Figure is available in color online only. 

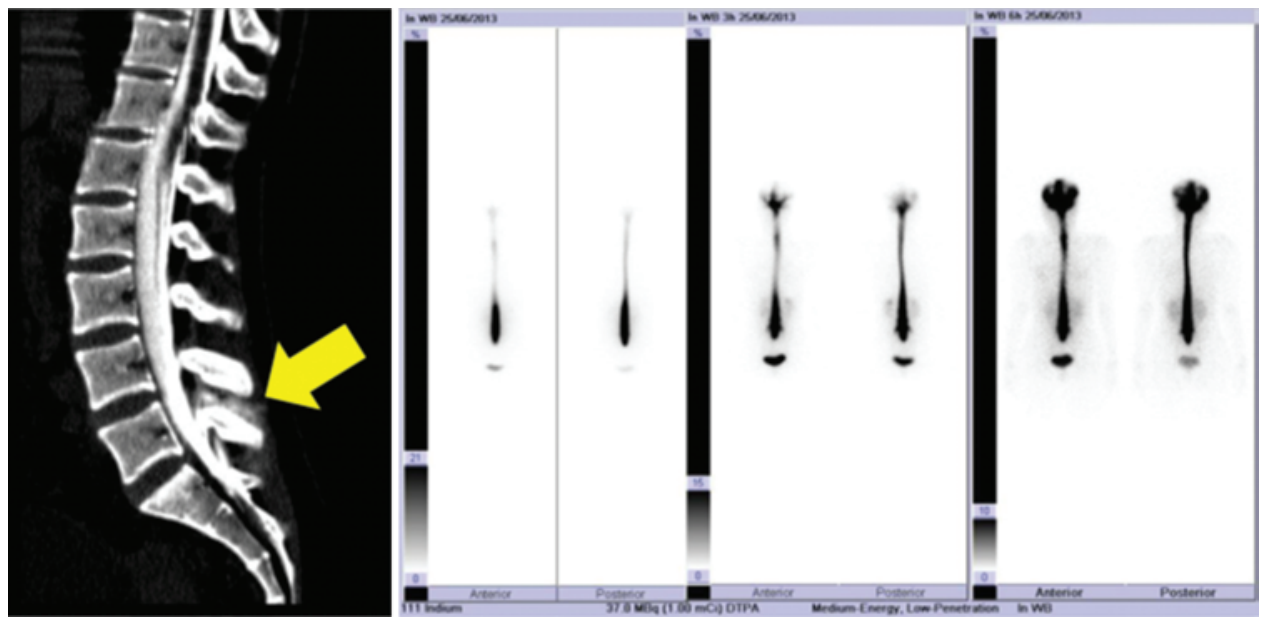

FIG. 3. Left: Leakage was observed in the sagittal CTM (yellow arrow). Right: Leakage was not observed in the sagittal RIC. Figure is available in color online only.
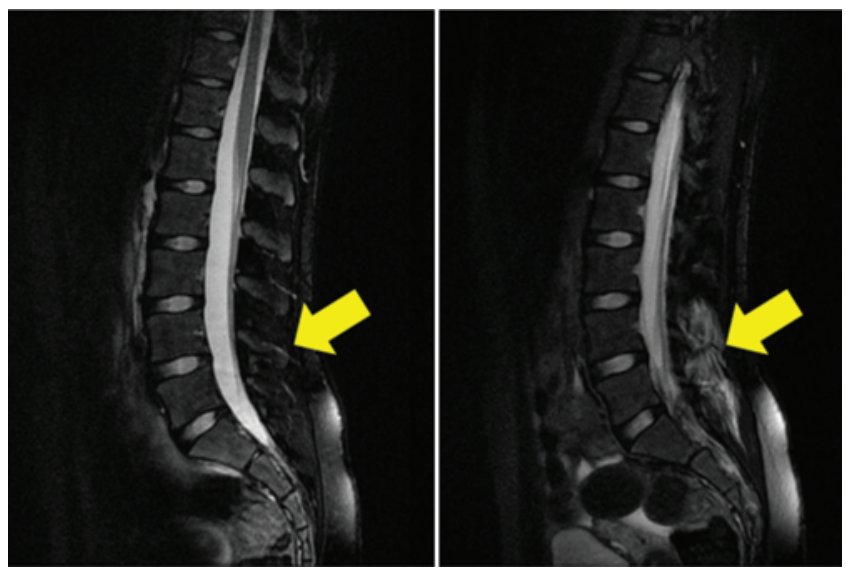

FIG. 4. Left: No finding was observed in the sagittal lumbar MR image before injection (yellow arrow). Right: Leakage was observed in the lower lumbar lesion in the sagittal lumbar MR image after injection (yellow arrow). Figure is available in color online only. lumbar spine (Fig. 4 right). Using a sterile technique, 20 $\mathrm{ml}$ of autologous blood was injected directly into the epidural space. Injection of blood was stopped because of the occurrence of numbness in both lower limbs. The patient reported complete symptomatic relief without any recurrence. Numbness was improved at follow-up of 5 months.

\section{Case 3}

A 72-year-old woman was referred to our hospital with a 3-month history of progressively worsening headache and neck pain. Her CSF pressure was $2 \mathrm{~cm} \mathrm{H}_{2} \mathrm{O}$ at presentation. The leakage site was not identified by CTM or RIC (Fig. 5). The leakage site was identified in C2-3 by an FST2WSI after saline infusion (Fig. 6 right). Bilateral chronic subdural hematomas (CSDHs) were confirmed on the CT images. Natural healing of subdural hematomas by complete recovery of CSFH had been reported previously; therefore, we prioritized treatment of the CSFH. ${ }^{4}$ Using a sterile technique, $10 \mathrm{ml}$ of autologous blood was injected into the epidural space. However, the patient's state of consciousness deteriorated the next day. We speculated that the improvement of CSFH caused the increased intracranial pressure. The patient's state of consciousness was im-
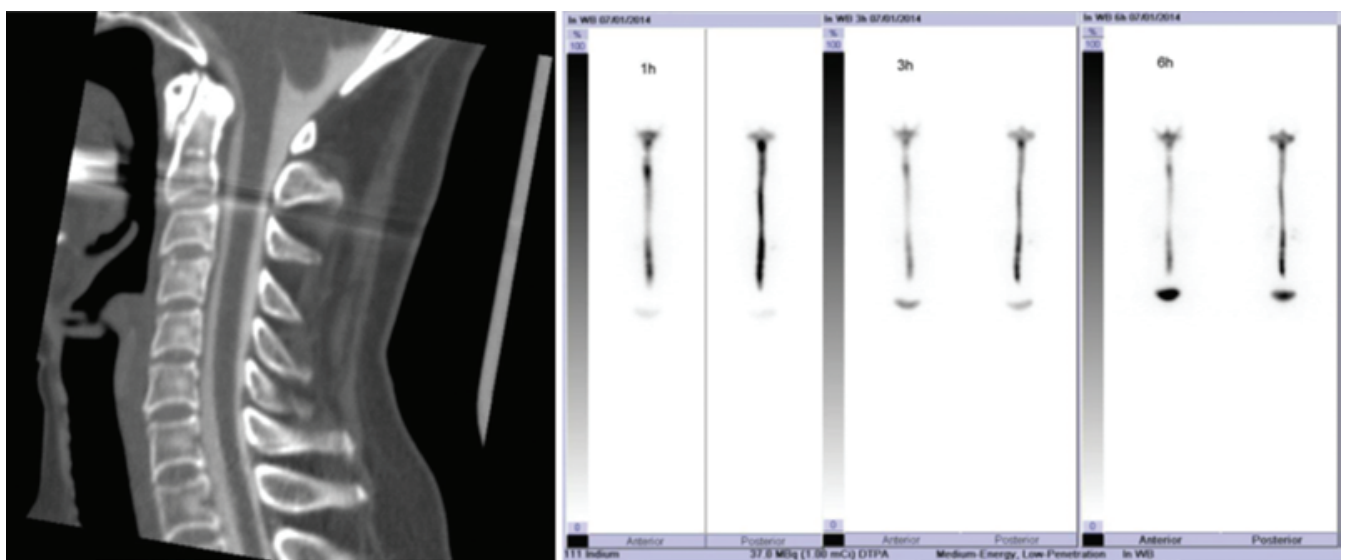

FIG. 5. Left: Leakage was not observed in the sagittal CTM. Right: Leakage was not observed in the sagittal RIC. Figure is available in color online only. 

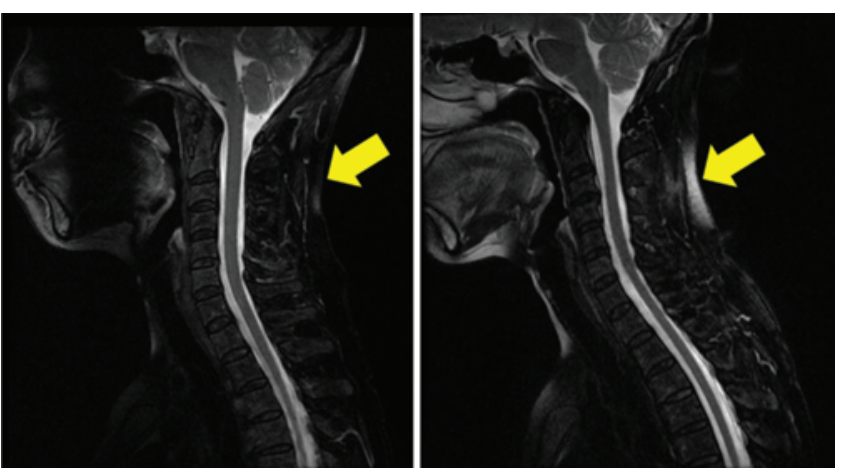

FIG. 6. Left: No finding was observed in the sagittal cervical MR image before injection (yellow arrow). Right: Leakage was observed between $\mathrm{C}-2$ and $\mathrm{C}-4$ in the sagittal cervical MR image after the injection (yellow arrow). Figure is available in color online only.

proved by emergency bur-hole surgery. Thus, the patient reported complete symptomatic relief without any recurrence at follow-up of 3 months.

\section{Discussion}

The CSF leakage site could be confirmed in all 3 cases by FST2WSIs after saline infusion. The leakage site was confirmed by the usual modality, CTM, in only 1 (Case 2 ) of the 3 cases. The FST2WSIs obtained after saline infusion were clear enough to effectively discriminate the precise leakage site. All cases were cured completely in a single treatment by the EBP to the exact leakage site. There was no complication during procurement of FST2WSIs after saline infusion. The EBP caused transient numbness in 1 case and disturbed consciousness due to bilateral CSDHs.

We speculated that CSF overflowed from the leakage site owing to a large-volume infusion of saline. During the procedure, reassessment of the CSF pressure was the key factor in safely achieving a successful FST2WSI. On the other hand, in the usual modalities, the agent is diluted before flowing out to the epidural space, resulting in the blurred images characteristic of CTM and RIC.

The leakage volume of CSF in CSFH is often sustained in small amounts. Therefore, detection of the leakage site by MRI has been difficult. Large-volume infusion of saline while monitoring CSF pressure appears to have solved this problem. Obtaining FST2WSIs after saline infusion is a cost-effective method with no risk of allergy and radioisotope exposure.

This technical report has some limitations. The number of cases is small, and the follow-up period was short. We plan to treat more patients with CSFH with this new modality and perform follow-ups over a longer period.

\section{Conclusions}

We have presented a new modality for detecting the specific CSF leakage site: FST2WSIs obtained after saline infusion. This new modality allowed us to perform more effective EBP by providing information that was more precise. Because the validity of artificial CSF has been reported, ${ }^{2}$ we plan to change the saline to improve the method in the near future.

\section{References}

1. Buvanendran A, Byrne RW, Kari M, Kroin JS: Occult cervical (C1-2) dural tear causing bilateral recurrent subdural hematomas and repaired with cervical epidural blood patch. J Neurosurg Spine 9:483-487, 2008

2. Doi K, Kawano T, Morioka Y, Fujita Y, Nishimura M: Various irrigation fluids affect postoperative brain edema and cellular damage during experimental neurosurgery in rats. Surg Neurol 66:565-572, 2006

3. Mehta B, Tarshis J: Repeated large-volume epidural blood patches for the treatment of spontaneous intracranial hypotension. Can J Anaesth 56:609-613, 2009

4. Mitsuteru S, Satoshi N, Yumiko W, Hajime T: Spontaneous cerebrospinal fluid hypovolemia complicated by cerebral venous thrombosis and a chronic subdural hematoma. Japanese J Stroke 33:430-437, 2011

\section{Disclosure}

The authors report no conflict of interest concerning the materials or methods used in this study or the findings specified in this paper.

\section{Author Contributions}

Conception and design: Nakai, Takemura, Nonaka, Kawanishi. Acquisition of data: Nakai. Analysis and interpretation of data: Nakai, Masahira. Drafting the article: Nakai. Critically revising the article: Nakai. Reviewed submitted version of manuscript: Nakai. Approved the final version of the manuscript on behalf of all authors: Nakai. Statistical analysis: Nakai. Administrative/technical/material support: Nakai, Ueba. Study supervision: Nakai, Ueba.

\section{Correspondence}

Eiichi Nakai, Department of Neurosurgery, Kochi Medical School, Kochi University, Kohasu, Okoh-cho, Nankoku, Kochi 783-8505, Japan.email: enakai@kochi-u.ac.jp. 